\title{
Harmonic Evaluation of Traction System by Monte Carlo Simulation
}

\author{
YE Zhongming, PONG M. H., LO W. C., YUEN K. H. \\ Dept. of Electrical \& Electronics Engineering \\ Hong Kong University, Hong Kong
}

\begin{abstract}
This paper presents a method to predict the harmonic current level of traction system with phase-controlled DC Drives by Monte Carlo simulation. Based on Behavioral Modeling Technique (BMT), a model for electrical unit of traction is proposed. The probability density functions (pdf) of speed and notch numbers are obtained from longtime field measurement. The mean and variance of harmonic current of single electrical unit is obtained based on the speed pdf and traction electrical unit model. The results of Monte Carlo simulation are in good accordance with the experimental and analytic conclusions. The harmonics of a different number of trains are systematically investigated. It is shown the Total Harmonic Distortion (THD) decreases with the increase of the number of trains and the harmonic current per train decreases with the train number because of the harmonic cancellation.
\end{abstract}

Keywords: Harmonics, Probability, Traction System, Monte Carlo Simulation

\section{INTRODUCTION}

The electrification system of the Hong Kong railway is $25 \mathrm{KV}, 50 \mathrm{~Hz}$ single-phase current collection catenary system with electrified railway being fed from two track-side feeder stations. In each feeder station, duplicated single-phase supplies are taken from different phases of a local electrical power system via a $132 / 25 \mathrm{KV}$ transformer connected in open-delta. The traction motors get power through the transformers (Fig. 1) installed at the bottom of the trains and are energized from the overhead line through the pantograph. Phase-controlled thyristor rectifiers of traction electrical unit, which are the major nonlinear loads of the traction system, convert the secondary output of the transformer into DC current to supply the motors. In each such electrical unit, two identical rectifier bridges are connected in series, and are connected to the two secondary windings of equal voltages. Changing of the current of DC motor can be achieved by changing the firing angle of the controlled rectifier. Four notches are available to control the speed of the traction. For notch 1 and 2, the maximum speeds are $20 \mathrm{Km} / \mathrm{hr}$ and 40 $\mathrm{Km} / \mathrm{hr}$ respectively and in these notches, only the upper rectifier is put into operation. For notch 3 and 4, the maximum speed is 60 and $120 \mathrm{Km} / \mathrm{hr}$ respectively, while the lower rectifier is also put into operation.

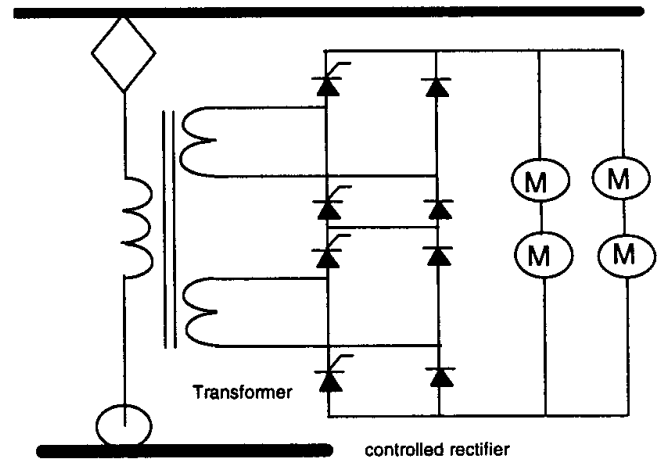

Fig. 1. The diagram scheme of the traction electrical unit

As it is well known, phase-controlled rectifier deteriorates power quality of the distribution system with heavy harmonic current and reactive power. Harmonics evaluation is important for both railway company and electrical power company. Compared with other power distribution system, the harmonic evaluation of the traction power supply system is even complicated owing to the fact that electrical units of trains produce different harmonics at different operation conditions (speed, loading, etc.), and the circuit parameters and topology of the overhead lines are time-dependent.

To simplify the evaluation of current harmonics level of the traction power system, we propose a method based on Monte Carlo simulation. A Behavioral Modeling Technique (BMT) is proposed for traction electric unit modeling which involves experiment data collection, model modification and evaluation. With probability density function of the speed of trains and the harmonic model of traction electrical unit, Monte Carlo simulation of traction system is carried out. The probability density function of current harmonics for a single train as well as sum harmonics at substations are studied. Conclusions are drawn at the end of the paper.

\section{MODELING OF ELECTRICAL UNIT \& TRACTION SYSTEM}

The modeling of the traction system consists of the following three modules: POWER SYSTEM module, TRACTION ELECTRICAL MODULE UNIT (EMU) 
module and TRACTION NET module. The scheme is plotted in Fig. 2. The electrical unit module calculates the harmonic current of the electrical unit at a given operation mode, speed, loading and line voltage. The TRACTION NET module specifies the location of individual train and the POWER SYSTEM module calculates the line voltage given the impedance matrix of the electric network and line currents.

The traction electrical unit is a typical phase controlled thyristor rectifier with $\mathrm{DC}$ drive load. The reactive and harmonic current are determined by the firing angle. For small firing angle, the harmonic current is low in magnitude, but the distortion ratio is serious. For large firing angle, the harmonic current is high but the current distortion is relatively low.

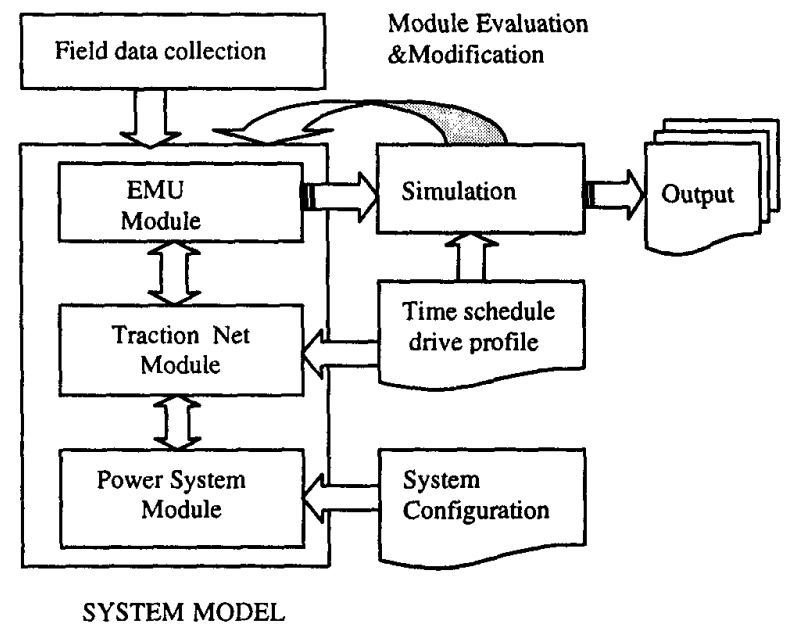

Fig 2. The diagram scheme of the traction system modeling

With BMT method, the electrical unit of the traction is treated as black box. Tests for train characteristic are performed for various operation conditions, i.e., motoring, coasting, braking and parking for different speeds and notches. The measured data of the waveforms are converted to frequency domain by Fast Fourier Transform Algorithm. As far as the practical system is concerned, decomposition in frequency up to 13 rd harmonics is sufficient for the purpose of traction system. For each order of harmonics, it can be represented as two dimensional variables in real-imaginary projections:

$$
\begin{aligned}
& I_{h}(v, n)=X_{h}(v, n)+j Y_{h}(v, n) \text { for } h=1,2,3, \ldots \\
& X_{h}=X_{h}(v, n) \\
& Y_{h}=Y_{h}(v, n)
\end{aligned}
$$

where $X_{h}, Y_{h}$ are the real and imaginary part of the $h$ th harmonic components and respectively $v, n$ stand for speed and notch number respectively. Since these components of current are changing from speed to speed, from notch to notch, they are represented as notch and speed dependent variables.

To get all current waveforms at all speeds is impossible, since the traction electrical unit controls the traction speed continuously from zero to maximum of the possible speed. Only the current waveforms of a few discrete speed samples are measured and analyzed. However, in order to simulate the system, frequency representation for all speeds are necessary. This can be done by interpolation in frequency domain since the harmonics components changes smoothly with speed as long as notch number keeps the same. By interpolating, a mapping between the speed and the harmonic components of currents is established. Fig.3 shows typical curves of amplitude and phase angle of $3^{\text {rd }}, 5^{\text {th }}, 7^{\text {th }}$ harmonics and fundamental current changing with speed during motoring. It is evident that if trains run at different speeds, there will be cancellation of harmonics, since the phase angles are different.
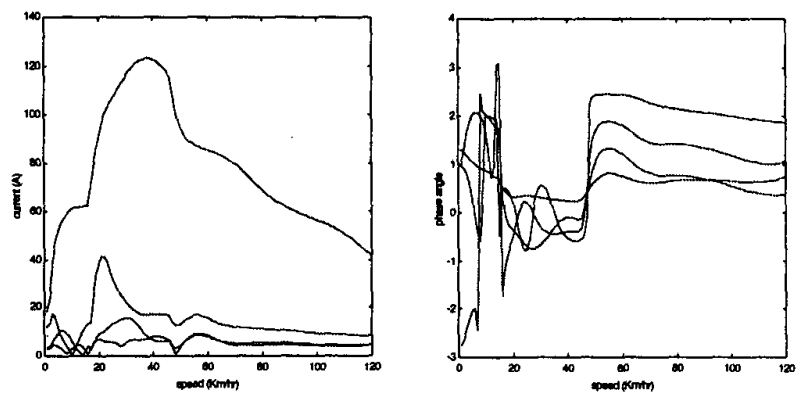

Fig. 3. The fundamental and 3,5,7 order harmonic current versus speed during motoring (from top: $1 \mathrm{st}, 3 \mathrm{rd}, 5 \mathrm{th}$ and $7 \mathrm{th}$ )

The modeling of the traction net system is achieved by building up a set of tables including the schedule of the trains running in the system and the overhead line connections and the locations of the stations. The power system is modeled in frequency domain as linear system. Whether or not the long-line effects will be included in the model depends on both the line length and the frequency interested. In this study of urban railway transmission system, the line is not longer than $20 \mathrm{Km}$, and the concerned harmonics are not higher than $19^{\text {th }}$, the line impedance can be omitted.

\section{SPEED PROBABILITY DENSITY FUNCTION}

Since the current harmonics are determined by the operation modes especially the speed and notch number, these 
two parameters can be selected as random variables for system harmonics probabilistic analysis. Based on the statistic knowledge of speed and notch number, Monte Carlo simulation can be used to evaluate the harmonics statistics of the system. According to probability theory, the probability of certain event can be approximated by the frequency of the event. Thus an automatic data logging system is developed to measure the speed, notch number of a typical train in the traction system, together with the traction electrical unit current. With a measurement lasting for two weeks, the probability density functions of the train during motoring are obtained and shown below in Fig. 4. The speed resolution of the measurement is $0.5 \mathrm{Km}$ per hour. The pdf of speed for all operation modes can also be obtained.

$p_{v}$ is used to denote the probability density for speed $v$. The pdf of speed during motoring is a function of both speed and notch number, from the above to the bottom in Fig. 4 are notch 1, 2, 3 and 4 accordingly.

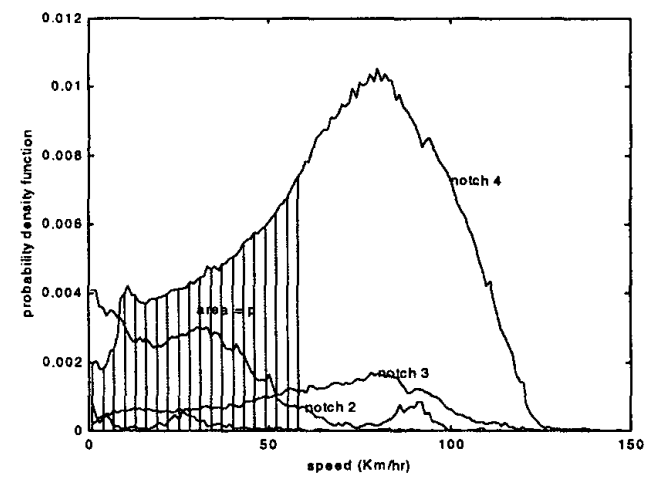

Fig. 4. The probability density function of speed for motoring operation only (from bottom to top: notch 1,2,3 and 4).

The probability density function curves are divided to every $\mathrm{Km} / \mathrm{hr}$ per step, that is the scale of speed is $v_{1}, v_{2}, \ldots, v_{150} . \Delta v=1 K m / h r$.

The probability of the speed $v_{i}$ with notch number $n_{j}$ is defined as:

$$
P_{v_{i}, n_{j}}=P\left(v_{i} \leq V<v_{i+1}, \text { notch }=n_{j}\right)
$$

then it can be calculated from the probability density function:

$$
P_{v_{i}, n_{j}}=\frac{\left(p_{v_{i}, n_{j}}+p_{v_{i+1}, n_{j}}\right)}{2} \Delta v
$$

\section{HARMONIC CURRENT OF INDIVIDUAL TRACTION ELECTRICAL UNIT}

In many simulation tools, Monte Carlo simulation is integrated into the whole software, like PSPICE. The idea is just like that: assume the distribution of some key parameters of the system are known, generate the random values of the parameters in such a way that the samples will still follow the probability distribution, and use the simulation software to get the samples of the variables concerned, such as loss, efficiency, power factor, etc. Therefore, the probability distribution of the variables can be obtained. Usually, the distribution of the random samples of the parameters of the system is simplified to the form we are well known, and can be generated by computer mathematically.

In order to use Monte Carlo method to the traction model, firstly random samples of speed which have the same pdf as the practical situation should be generated. To do this, we convert the pdf of speed to the cumulative form as shown in Fig. 5. Then the cumulative probability to speed and notch number is point to point mapped, thus by generating the probability from 0 to 1 with unity distribution, we get the corresponding random speed samples with almost the same pdf as the one we already get. Based on the random speed samples obtained, Monte Carlo simulation can be performed. And the mean and variance of harmonic current of individual traction electrical unit during motoring are given in Table I with 30,000 samples.

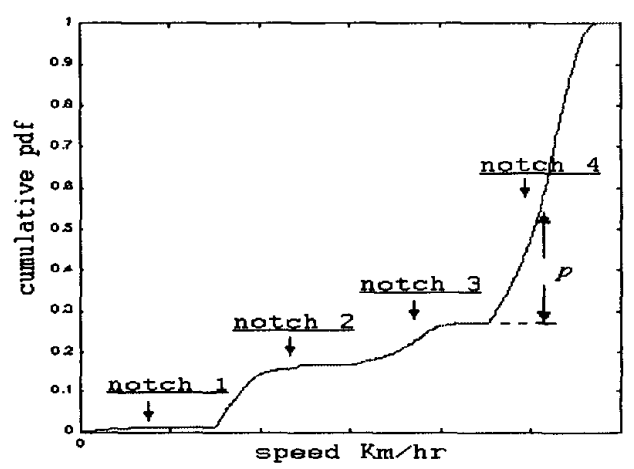

Fig. 5. The corresponding cumulative pdf of speed.

The real part of harmonics of order $h$ for a single electrical unit can be theoretically evaluated from the following

$$
\begin{aligned}
\widetilde{\mu_{x_{h}}} & =\sum_{n \text { otch }}^{4} \int X_{h}(v, n) p_{v, n} d v \\
& =\sum_{\text {notch }}^{4} \sum_{v=0}^{\text {Maxspeed }} X_{h}(v, n) P_{v, n}
\end{aligned}
$$

The variance of the real part of harmonics components is shown:

$$
\widehat{\sigma_{X_{h}}}=\sqrt{\sum_{n o t c h}^{4} \sum_{v=0}^{\text {Maxspeed }} X_{h}(v, n)^{2} P_{v, n}-\left(\widehat{\mu_{x_{h}}}\right)^{2}}
$$


Similarly, we get the formula to calculate the imaginary part:

$$
\widehat{\mu_{Y_{h}}}=\sum_{n o t c h}^{4} \sum_{\nu=0}^{\text {Maxspeed }} Y_{h}(v, n) P_{v, n}
$$

and the variance of the current imaginary part:

$$
\widetilde{\sigma_{Y_{h}}}=\sqrt{\sum_{\text {notch }}^{4} \sum_{\nu=0}^{\text {Maxspeed }} Y_{h}(\nu, n)^{2} P_{v, n}-\left(\widehat{\mu_{Y_{h}}}\right)^{2}}
$$

The mean and variance of harmonics for individual traction electrical unit during motoring are calculated by these two approaches and are compared in Table I. High agreement is observed which indicates the reliable of the Monte Carlo simulation approach with 30,000 samples.

TABLE I

THE MEAN AND VARIANCE OF CURRENT HARMONICS OF INDIVIDUAL TRACTION ELECTRICAL UNIT DURING MOTORING BY ANALYTIC METHOD AND MONTE CARLO SIMULATION

(I - by analytic method, II - by simulation)

\begin{tabular}{|c|c|c|r|r|r|r|r|r|r|}
\hline & \multicolumn{3}{|c|}{$\begin{array}{c}\text { magnitude of } \\
\text { harmonics (A) }\end{array}$} & \multicolumn{3}{c|}{$\begin{array}{c}\text { real part of } \\
\text { current (A) }\end{array}$} & \multicolumn{3}{c|}{$\begin{array}{c}\text { imaginary part } \\
\text { current (A) }\end{array}$} \\
\hline & mean & var & \multicolumn{2}{|c|}{ mean } & var & \multicolumn{2}{c|}{ mean } & var \\
\hline & I & II & I & I & II & I & I & II & I \\
\hline lst & 70.9 & 70.8 & 28.7 & 57.6 & 57.3 & 28.7 & 38.9 & 38.9 & 14.1 \\
\hline 3rd & 12.3 & 12.3 & 6.6 & 8.25 & 8.21 & 6.74 & 5.14 & 5.26 & 7.51 \\
\hline 5th & 5.66 & 5.64 & 2.99 & 1.49 & 1.46 & 3.92 & 3.39 & 3.43 & 3.45 \\
\hline 7 th & 4.44 & 4.46 & 2.12 & -1.15 & -1.19 & 3.66 & 2.33 & 2.34 & 2.47 \\
\hline 9th & 3.69 & 3.71 & 1.46 & -2.1 & -2.14 & 3.07 & -0.36 & -0.36 & 1.34 \\
\hline 11 th & 2.62 & 2.62 & 1.01 & 0 & 0 & 1.56 & -1.59 & -1.59 & 1.7 \\
\hline 13 th & 1.53 & 1.53 & 0.66 & 0.55 & 0.55 & 0.99 & 0.2 & 0.19 & 1.21 \\
\hline
\end{tabular}

\section{Monte Carlo Simulation Of N TRains}

When considering the harmonics of a traction substation with $\mathrm{N}$ trains, the impedance of power line can be ignored, since the lines are very short. The harmonics current at substation can be represented as vectorial sum of the harmonics phasors of $\mathrm{N}$ trains in frequency domain, that is :

$$
I_{h}=\sum_{j=1}^{N} X_{j, h}+j \sum_{j=1}^{N} Y_{j, h} \text { for } h=1,2,3, \ldots
$$

Assume the operation conditions of $\mathrm{N}$ trains are independently random. When trains are parked in railway stations or in traction factory for maintenance, it generates little harmonic current, therefore only moving trains are considered. Based on the speed pdf during motoring, the harmonics current and Total Harmonic Distortion (THD) are investigated for 8 trains which have similar speed pdf as Fig. 4. The pdf of the sum current harmonics and fundamental component at substation are plotted in Fig. 6, 7, 8 and 9 respectively.

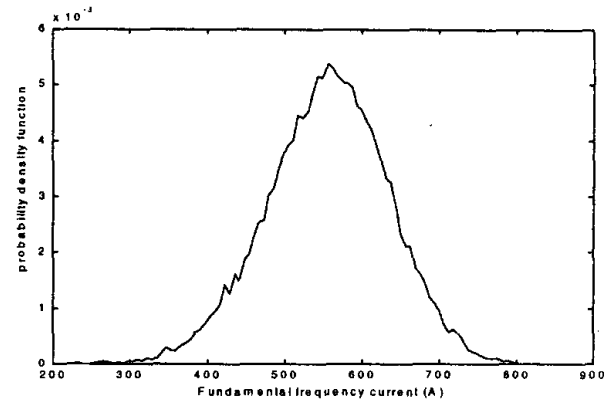

Fig. 6. The probability density function of fundamental component.

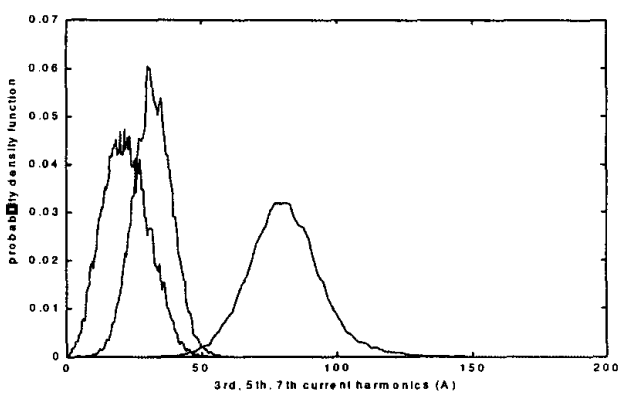

Fig. 7. The pdf of harmonics $3 \mathrm{rd}, 5$ th, 7 th for the sum current of 8 trains (from right to left: 3 rd, 5 th, 7 th).

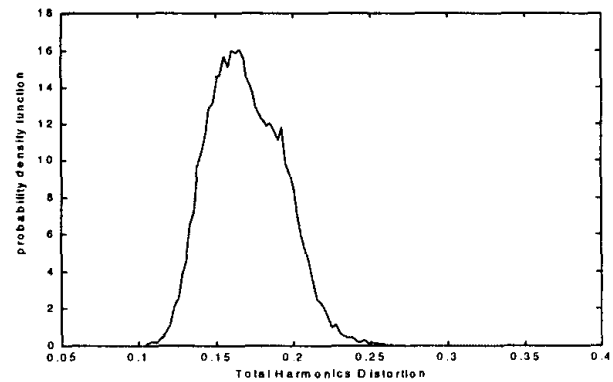

Fig. 8. The pdf of THD of the sum current of 8 trains

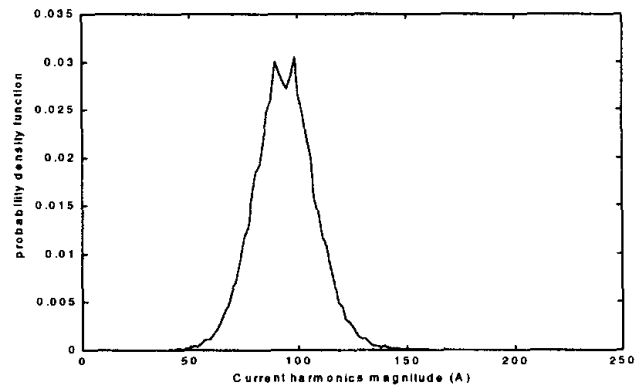

Fig. 9. The probability density function of the sum harmonic current of 8 trains. 
From the probability density functions, it is evident that the harmonics per train when 8 trains are running is lower than that of a single train. This is caused by the harmonic phasorial cancellation. Since individual current harmonics are determined by the speed, operation mode of individual train, the probability for all trains to run at the same speed and with the same operation mode is rare. Thus the probability of current harmonics of different trains to have similar magnitude and phase angle is very low. More often, the phase angle of each current harmonics from different trains are different. Therefore harmonic cancellation is always the case. For low order harmonics, the cancellation is not so evident as for high order harmonics. For instance, the fundamental current per train for 8 trains is almost the same as that of a single train, while the $13 \mathrm{rd}$ harmonic component per train has a reduction of about 33 percent.

Applying the Monte Carlo simulation to the case of $2,3, \ldots$, up to 10 trains, we find the mean value for the harmonics per train decrease with the increase of train number. Since the increase of train number will increase the randomness of the phase angle of individual harmonics, the probability for harmonics cancellation increases. In Fig. 10, 11 and 12, the mean of 3rd and 13rd order harmonics per train are plotted against train number. 20,000 samples are simulated.

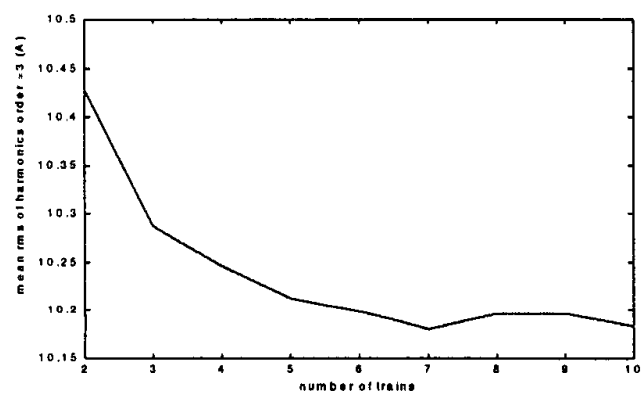

Fig. 10 The mean value of 3 rd order harmonics magnitude versus train number.

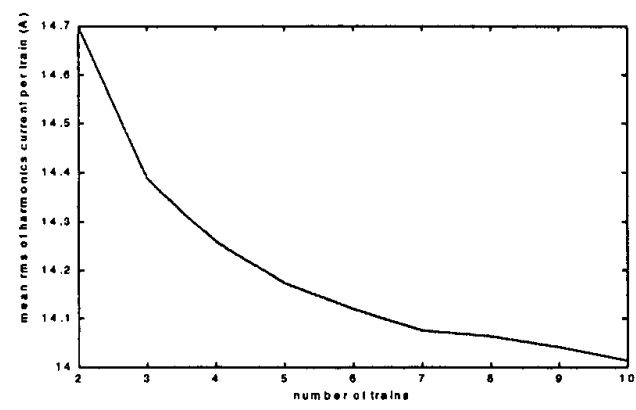

Fig. 12. The mean harmonic current magnitude versus different train number.

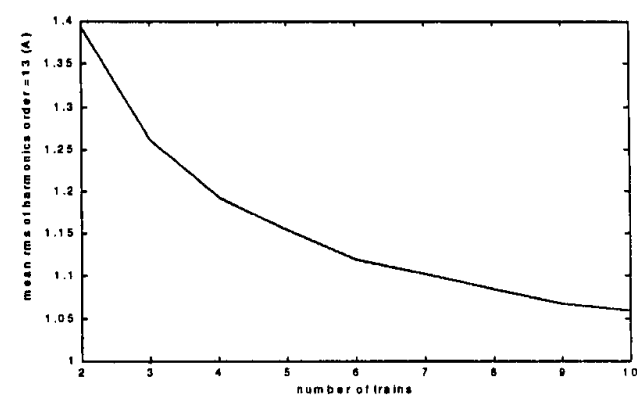

Fig. 11. The mean magnitude of 13 rd order harmonics versus different train numbers.

\section{THE HARMONICS AT SUBSTATIONS FOR ONE DAY}

The daily current harmonics at power feeding substations can be an important parameter during filter design stage (if power line filters are required). The above mentioned method can also be used here to determine the probability features of current harmonics at substation. In this analysis, the train number fluctuates with time, since the number of passengers varies through out the day. During rush hours, there are more trains in the system to meet the load requirement. In Hong Kong, the daily rush hours are 8:00-10:00 AM and 4:007:00 PM, which is demonstrated in Fig.13. This train number profile is drawn according to the time table of the local Traction Cooperation in Hong Kong. The time is quantized to 10-minute intervals, and assume at each instant of sampling the operation mode and speed of the trains are independent from each other. In this way the probability density function of harmonics and cumulative probability density function (cpdf) of harmonics current at feeding substation can be calculated on a daily basis and plotted in Fig. 14, 15 and 16, with variation of train number during a typical working day being taken into account. It should be noted that here the currents are referred to the currents before a capacitor filter bank.

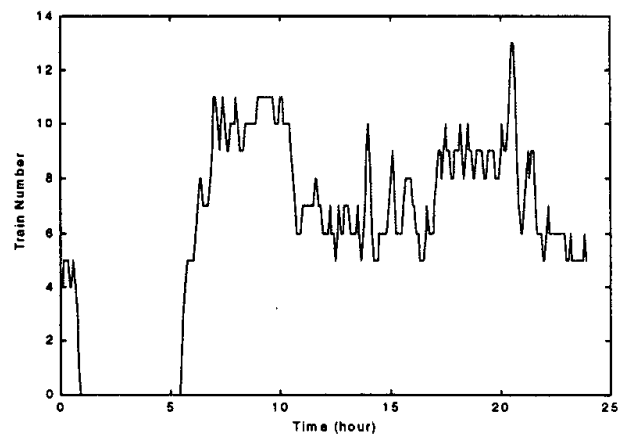

Fig. 13. The profile of the train number of Traction System in Hong Kong for a period of 24 hours. 


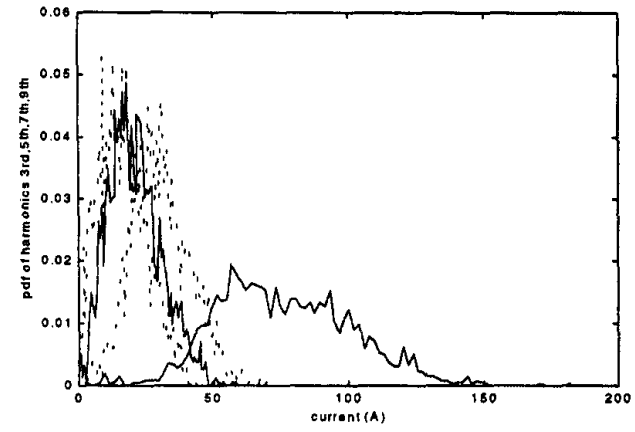

Fig. 14. The probability density function of harmonic current magnitude ( from right: $3 r d, 5 t h, 7 t h, 9 t h$ ).

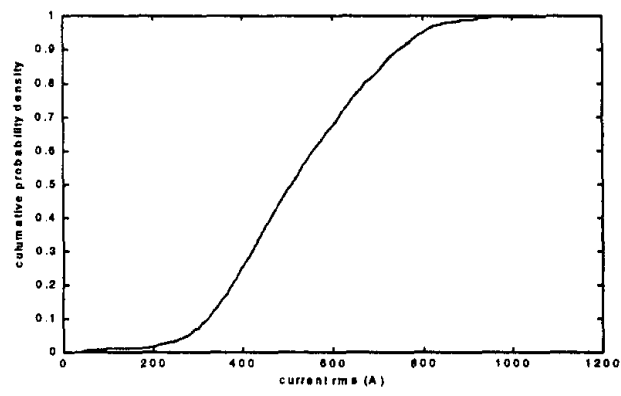

Fig. 15. The cumulative pdf of current magnitude at substation

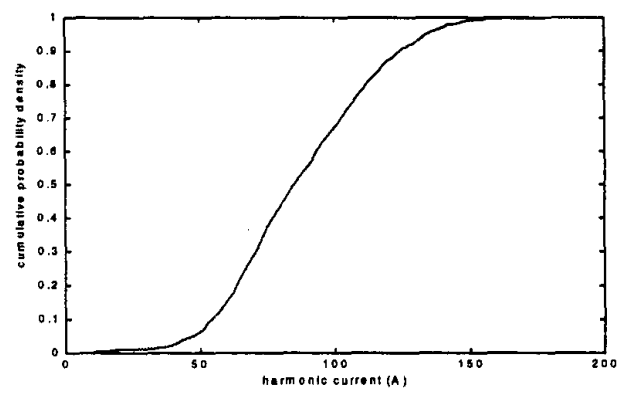

Fig. 16. The cumulative pdf of the harmonics current at substation

\section{CONCLUSION}

Harmonics Investigation of traction power system is presented based on Monte Carlo simulation method. An integrated traction system model is developed which consists of Behavioral Modeling Technique (BMT) based traction electrical unit module, power system module and traction net module. Probability density function of a single traction electrical unit as well as a number of trains are studied by
Monte Carlo simulation. It is shown harmonics current changes mainly with the number of trains that are in service in the traction system. But the Total Harmonic Distortion varies slightly during the working hours. The cancellation of harmonics is observed, but for different order of harmonics, cancellation can be different.

\section{ACKNOWLEDGMENT}

The authors would like to acknowledge the Kowloon \& Canton Railway Corporation (KCRC) in Hong Kong for their help and advice throughout this project.

\section{REFERENCES}

J. A . Orr, A. E. Emanuel, K. W. Oberg, Current harmonics generated by a cluster of electric vehicle battery chargers, IEEE Trans. Power System 101, No.3 March 1982, pp691-700.

D. Okelly, Probability characteristics of fundamental and harmnic sequence components of randomly varying loads, IEE-PROC., Vol. 129, Pt. C. No.2, March 1982, pp70-78.

R.E.Morrison, A. D. Clark, Probabilistic representation of harmonic currents in AC traction system, IEE PROC., Vol.131. Pt. B, No.5, Sept. 1984, pp181-189.

A. E. Emanuel, S. R. Kaprielian, Contribution to the theory of stochastically periodic harmonics in power systems, IEEE Trans. Power Delivery, Vol. PWRD-1, No. 3, July 1986, pp285-293.

Y. Baghzouz, Owen Tan, Probabilistic modeling of power system harmonics, IEEE Trans. Ind. Application, Vol. 23, No. 1, Jan. 1987, pp173-180.

W. Kazibwe, et al, EEE Trans. Power Delivery, Vol.4, No. 1, Jan. 1989, pp621-628.

Y. J. Wang, Lambert Pierrat, Probabilistic modeling of current harmonics produced by an AC/DC converter under voltage unbalance, IEEE Trans. Power Delivery, Vol. 8, No.4, 1994, pp2060-2066.

Y. J. Wang, Lambert Pierrat, Summation of harmonic currents produced by $A C / D C$ static converters with randomly fluctuating loads, IEEE Trans. Power Delivery, Vol. 9, No.2, April 1994, pp1129-1135.

S.R. Kaprielioan, A.E. Emanuel, R. Dwyer, H. Melita, Predicting voltage distortion in a system with multiple random harmonic sources, IEEE Trans. Power Delivery, Vol. 9, No.3, July 1994, pp1632-1638.

P. Staats, W. Grady, A. Arapostathis, R. Thallam, A statistical method for predicting the net harmonic currents generated by a concentration of electric vehicle battery chargers, IEEE Trans. Power Delivery, Vol.12, No.3, July 1997, pp1258-1266

K. H. Yuen, M. H. Pong, W. C. Lo, Z. M. Ye, Modeling of electric railway vehile for harmonic analysis of traction power supply system using spline interpolation in frequency domain, IEEE APEC 1999, in press. 